\title{
Magnetic Resonance Imaging of the Prostate in the PI-RADS Era
}

\author{
Bernd Hamm and Patrick Asbach
}

\section{Learning Objectives}

- To understand the current role of prostate MRI for imaging-based targeted prostate biopsy, staging, and active surveillance

- To learn how to optimize the multiparametric prostate MR imaging protocol

- To illustrate how to use the PI-RADS classification for prostatic lesion assessment

- To understand the need of structured reporting in communicating with the urologists

- To discuss the most common pitfalls in multiparametric MRI of the prostate

\subsection{Introduction}

Over the past years, continuing technical innovation in combination with a broad research activity has resulted in a high diagnostic accuracy of magnetic resonance imaging (MRI) of the prostate. At the same time, the Prostate Imaging Reporting and Data System (PI-RADS) has standardized image acquisition and reporting and facilitated the communication of imaging findings to the urologist and can therefore be considered an obligatory key element in prostate MRI. This has had a tremendous impact on the diagnostic workup of patients with suspected prostate cancer, with MRI being incorporated in multiple prostate cancer guidelines (e.g., NICE, AUA, German S3-Guideline) by now. As a direct result, imaging-based targeted prostate biopsy has increased almost at the same speed, and urologists not only heavily rely on accurate interpretation of MRI of the prostate

B. Hamm $(\bowtie) \cdot$ P. Asbach

Department of Radiology, Charité-Universitätsmedizin Berlin, Berlin, Germany

e-mail: bernd.hamm@charite.de but actively claim high-quality MRI scans for their daily practice because prostate MRI has direct impact on their cancer detection rate. Furthermore, a paradigm shift is taking place in the urological community regarding the care of lowgrade prostate cancer patients, where therapy is more and more replaced by active surveillance (AS). Prostate MRI plays an important role in AS not only during the initial assessment whether the patient is a candidate for AS but also during the surveillance of the disease.

Therefore, any abdominal and genitourinary radiologist if not already performing prostate MRI will subsequently be involved, and we forecast prostate MRI to be an indispensable diagnostic step for any patient with a clinical suspicion for prostate cancer or during surveillance of low-grade prostate cancer in the near future.

\subsection{PI-RADS}

The European Society of Uroradiology (ESUR) has introduced PI-RADS (Prostate Imaging Reporting and Data System) in 2012. An updated version (PI-RADS v2) was published in 2015 in collaboration with the American College of Radiology (ACR) and the AdMeTech Foundation. PI-RADS is not based on evidence from clinical research trials rather than on expert knowledge; however, several studies have confirmed that the PI-RADS system improves the diagnostic accuracy of mpMRI. The overall rationale for implementation of PI-RADS was to "improve detection, localization, characterization, and risk stratification in patients with suspected cancer in treatment naïve prostate glands." PI-RADS is currently not applicable to assess treatment response in prostate cancer patients. The following specific definitions and aims regarding MR imaging and reporting are targeted by PI-RADS: 


\subsubsection{Clinical Considerations}

Timing of mpMRI after prostate biopsy does not necessarily need to be postponed since clinically significant cancer is likely in a location not altered by post biopsy hemorrhage when the biopsy was negative. For local staging of prostate cancer, a delay of a minimum of 6 weeks might be advantageous. No specific patient preparation is necessary; however, the administration of a spasmolytic drug may be beneficial. Bowel cleaning is not recommended; however, the patient should evacuate the rectum if possible.

\subsubsection{Technical Considerations}

The field strengths of 1.5 and $3 \mathrm{~T}$ can both be adequate (even without an endorectal coil at $1.5 \mathrm{~T}$ ) when the scan parameters are tailored to small field-of-view imaging of the prostate and contemporary scanner technology is used (specifically multichannel phased-array surface coils and high-performance gradients); however, $3 \mathrm{~T}$ is the preferred field strength (higher signal-to-noise ratio, shorter scan time).

Multiparametric MRI of the prostate should include the following sequences:

T2-weighted imaging: 2D turbo spin-echo sequence, slice thickness $\leq 3 \mathrm{~mm}$ (no interslice gap), and in-plane spatial resolution $\leq 0.7 \mathrm{~mm}$ (phase-encoding direction) $\mathrm{x} \leq 0.4 \mathrm{~mm}$ (frequency-encoding direction).

Diffusion-weighted imaging (DWI): spin-echo EPI (echo planar imaging) sequence with fat saturation, slice thickness $\leq 4 \mathrm{~mm}$ (no interslice gap), in-plane spatial resolution $\leq 2.5 \mathrm{~mm}$ (phase- and frequency-encoding direction), and at least two $b$-values (low $b$-value of $50-100 \mathrm{~s} / \mathrm{mm}^{2}$ and a high $b$-value of $800-1000 \mathrm{~s} / \mathrm{mm}^{2}$ ) with consecutive calculation of an ADC map. If SNR allows, a very high $b$-value of 1400 $2000 \mathrm{~s} / \mathrm{mm}^{2}$ should be measured. An alternative is to calculate a high $b$-value, which is less SNR critical.

Dynamic contrast-enhanced (DCE) imaging: 2D or 3D gradient-echo sequence with a temporal resolution below $10 \mathrm{~s}$ (preferably below $7 \mathrm{~s}$ ) per acquisition, slice thickness $\leq 3 \mathrm{~mm}$ (no interslice gap), and in-plane spatial resolution $\leq 2 \mathrm{~mm}$ (phase- and frequency-encoding direction).

Slice orientation and slice thickness should match for all mpMRI sequences to allow side-by-side comparison. Also, a large field-of-view sequence covering the pelvic lymph nodes and the skeleton should be acquired.

\subsubsection{Assessment of Prostatic Lesions}

One major key element of PI-RADS is scoring the likelihood of a prostatic lesion to be clinically significant prostate cancer on a 5-point Likert-type scale (Table 11.1).
Since several different definitions of clinically significant prostate cancer exist, PI-RADS defines it as "Gleason score $\geq 7$ (including 3+4 with prominent but not predominant Gleason 4 component), and/or volume $\geq 0.5 \mathrm{cc}$, and/or extra prostatic extension (EPE)." The scoring system is based on typical imaging findings on the respective multiparametric MR sequence (exact definitions according to PI-RADS, see Tables 11.2, 11.3 and 11.4; for examples, see Figs. 11.1, 11.2, 11.3, 11.4, 11.5, 11.6, 11.7, $11.8,11.9,11.10,11.11,11.12$ and 11.13). For this purpose, typical examples for each score and sequence are included in the

Table 11.1 PI-RADS classification (5-point Likert-type scale)

\begin{tabular}{|l|l} 
PI-RADS 1 & $\begin{array}{l}\text { Very low (clinically significant cancer is highly } \\
\text { unlikely to be present) }\end{array}$ \\
PI-RADS 2 & $\begin{array}{l}\text { Low (clinically significant cancer is unlikely to be } \\
\text { present) }\end{array}$ \\
PI-RADS 3 & $\begin{array}{l}\text { Intermediate (the presence of clinically significant } \\
\text { cancer is equivocal) }\end{array}$ \\
PI-RADS 4 & $\begin{array}{l}\text { High (clinically significant cancer is likely to be } \\
\text { present) }\end{array}$ \\
PI-RADS 5 & $\begin{array}{l}\text { Very high (clinically significant cancer is highly } \\
\text { likely to be present) }\end{array}$
\end{tabular}

Table 11.2 T2-weighted imaging

\begin{tabular}{|c|c|c|}
\hline Score & Peripheral zone (PZ) & Transition zone (TZ) \\
\hline 1 & $\begin{array}{l}\text { Uniform hyperintense signal } \\
\text { intensity (normal) }\end{array}$ & $\begin{array}{l}\text { Homogeneous intermediate } \\
\text { signal intensity (normal) }\end{array}$ \\
\hline 2 & $\begin{array}{l}\text { Linear or wedge-shaped } \\
\text { hypointensity or diffuse mild } \\
\text { hypointensity, usually indistinct } \\
\text { margin }\end{array}$ & $\begin{array}{l}\text { Circumscribed hypointense } \\
\text { or heterogeneous } \\
\text { encapsulated nodule(s) } \\
\text { (BPH) }\end{array}$ \\
\hline 3 & $\begin{array}{l}\text { Heterogeneous signal intensity } \\
\text { or non-circumscribed, rounded, } \\
\text { moderate hypointensity, } \\
\text { includes others that do not } \\
\text { qualify as } 2,4 \text {, or } 5\end{array}$ & $\begin{array}{l}\text { Heterogeneous signal } \\
\text { intensity with obscured } \\
\text { margins, includes others } \\
\text { that do not qualify as } \\
2,4 \text {, or } 5\end{array}$ \\
\hline 4 & $\begin{array}{l}\text { Circumscribed, homogenous } \\
\text { moderate hypointense focus/ } \\
\text { mass confined to prostate and } \\
<1.5 \mathrm{~cm} \text { in greatest dimension }\end{array}$ & $\begin{array}{l}\text { Lenticular or non- } \\
\text { circumscribed, } \\
\text { homogeneous, moderately } \\
\text { hypointense, and }<1.5 \mathrm{~cm} \\
\text { in greatest dimension }\end{array}$ \\
\hline 5 & $\begin{array}{l}\text { Same as } 4 \text { but } \geq 1.5 \mathrm{~cm} \text { in } \\
\text { greatest dimension or definite } \\
\text { extraprostatic extension/ } \\
\text { invasive behavior }\end{array}$ & $\begin{array}{l}\text { Same as } 4 \text {, but } \geq 1.5 \mathrm{~cm} \text { in } \\
\text { greatest dimension or } \\
\text { definite extraprostatic } \\
\text { extension/invasive behavior }\end{array}$ \\
\hline
\end{tabular}

Table 11.3 Diffusion-weighted imaging (DWI)

Score Peripheral zone (PZ) or transition zone (TZ)

1 No abnormality (i.e., normal) on ADC and high $b$-value DWI

2 Indistinct hypointense on ADC

3 Focal mildly/moderately hypointense on ADC and isointense/ mildly hyperintense on high $b$-value DWI

$4 \quad$ Focal markedly hypointense on ADC and markedly hyperintense on high $b$-value DWI; $<1.5 \mathrm{~cm}$ in greatest dimension

5 Same as 4 but $\geq 1.5 \mathrm{~cm}$ in greatest dimension or definite extraprostatic extension/invasive behavior 


\section{Table 11.4 Dynamic contrast-enhanced (DCE) imaging}

\section{Score Peripheral zone (PZ) or transition zone (TZ)}

Negative No early enhancement or diffuse enhancement not corresponding to a focal finding on T2-weighted and/or DWI or focal enhancement corresponding to a lesion demonstrating features of $\mathrm{BPH}$ on $\mathrm{T} 2$-weighted imaging

Positive Focal and earlier than or contemporaneously with enhancement of adjacent normal prostatic tissues and corresponds to suspicious finding on T2-weighted and/or DWI
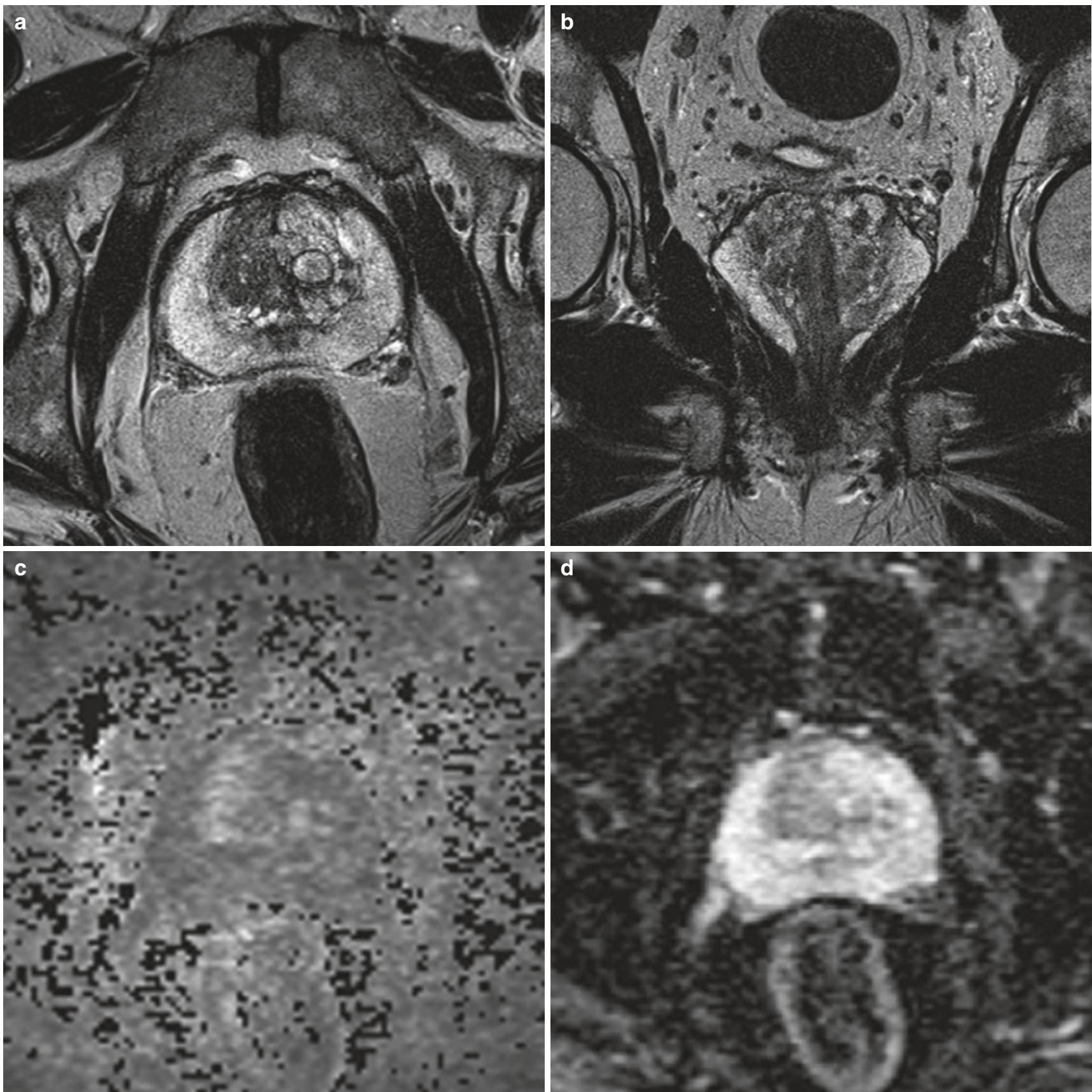

Fig. 11.1 (a-d) Normal peripheral zone. (a) Axial and (b) coronal T2-weighted sequence showing uniform hyperintense signal intensity of the peripheral zone (PI-RADS score 1). (c) Diffusion-weighted high
PI-RADS publication. The goal is to increase the diagnostic accuracy for detection of prostate cancer and to reduce the variability in image interpretation. Most preliminary studies report a good reader agreement which is higher for peripheral zone (PZ) lesions as compared to transition zone (TZ) lesions. Also, agreement is higher for PI-RADS score 4 and 5 compared to lower scores.

In the PI-RADS version 2, the diagnostic weighting of the multiparametric sequences has been changed compared to the first version. Now, a concept of a dominant sequence has 

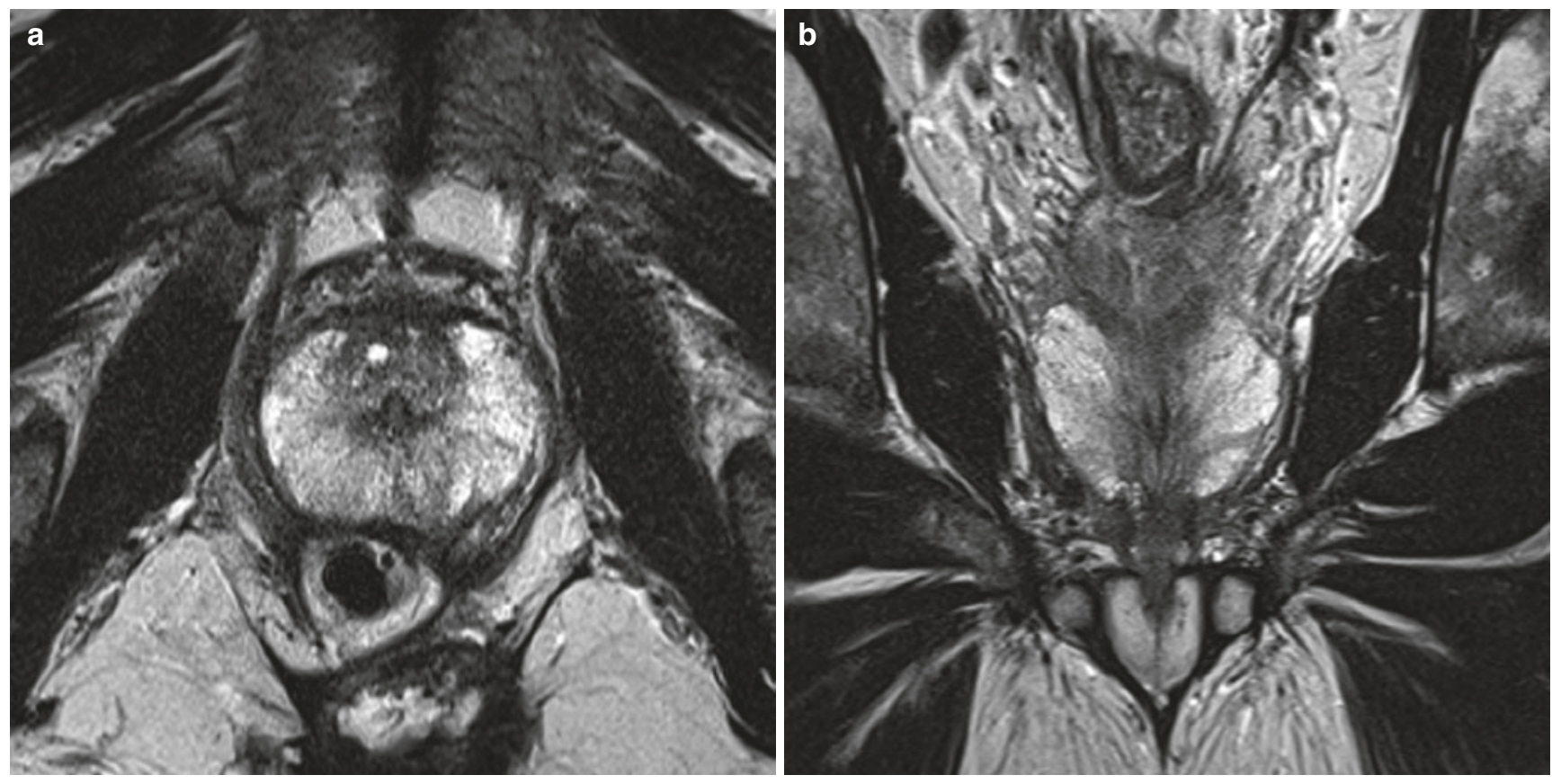

Fig. 11.2 (a, b) Normal transition zone. (a) Axial and (b) coronal T2-weighted sequence (dominant sequence for the transition zone) showing homogeneous intermediate signal intensity of the non-enlarged transition zone (PI-RADS score 1)

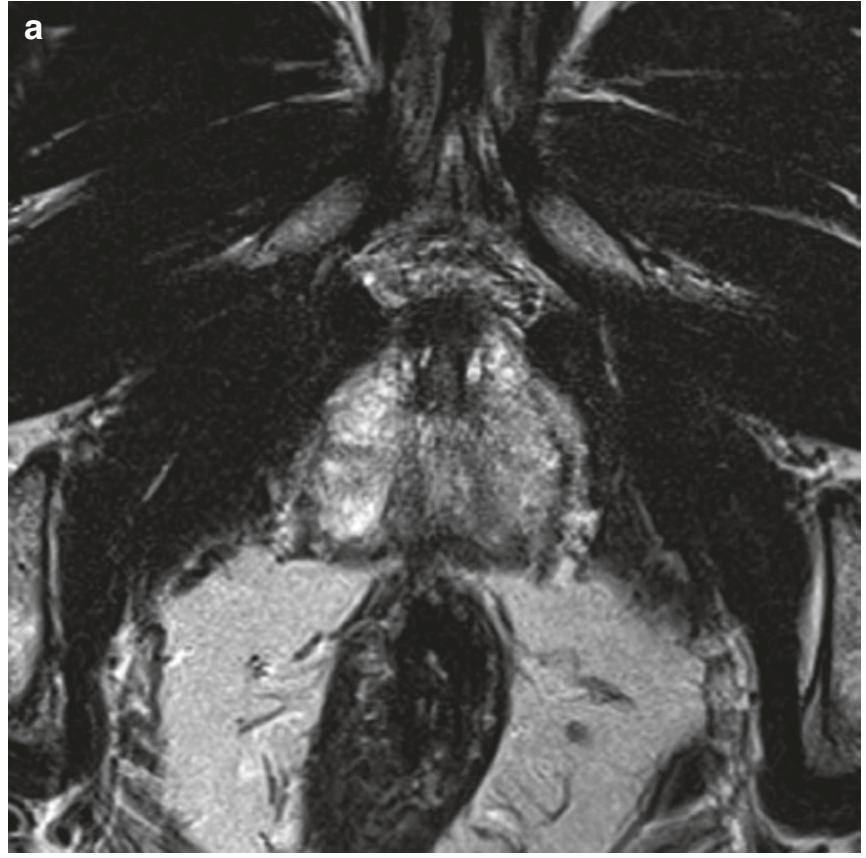

Fig. 11.3 (a-e) PI-RADS 2 changes of the peripheral zone. (a) Axial and (b) coronal T2-weighted sequence showing linear hypointensities in the bilateral peripheral zone (PI-RADS score 2). (c) Diffusionweighted high $b$-value (calculated $b=1400 \mathrm{~s} / \mathrm{mm}^{2}$ ) image shows no areas of increased signal intensity. (d) ADC map shows no focal hypointense areas (dominant sequence for the peripheral zone) in the

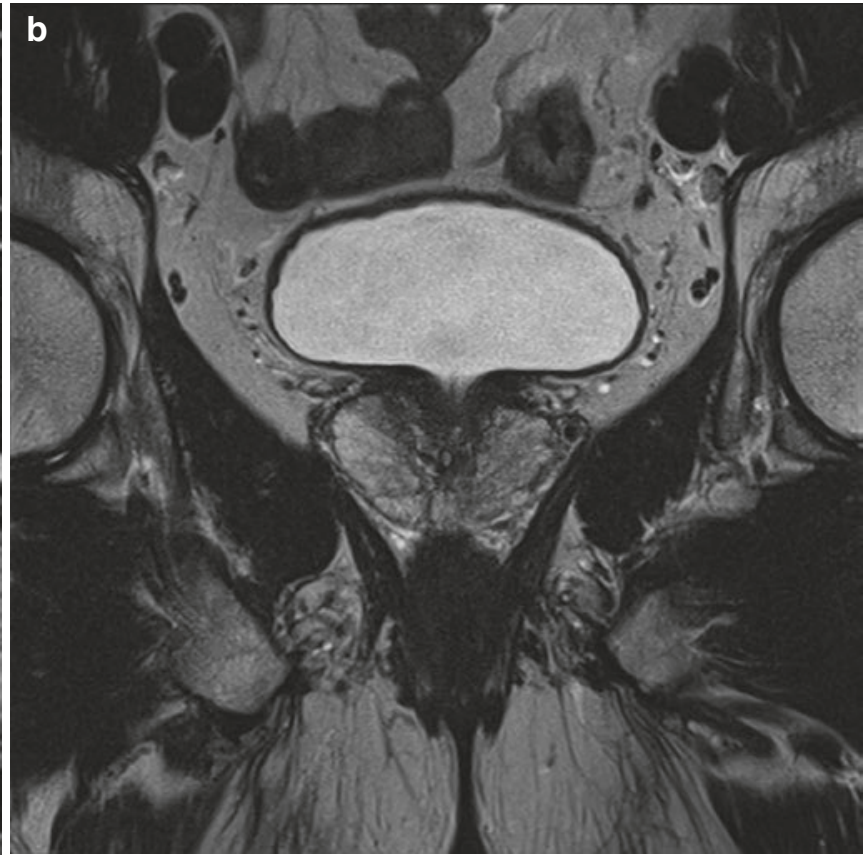

peripheral zone (PI-RADS score 2). (e) DCE sequence shows no focal or early enhancement (DCE negative) consistent with an overall PI-RADS score of 2. Linear T2 hypointensities in the peripheral zone are a frequent finding and may represent changes related to chronic prostatitis or post biopsy scarring 

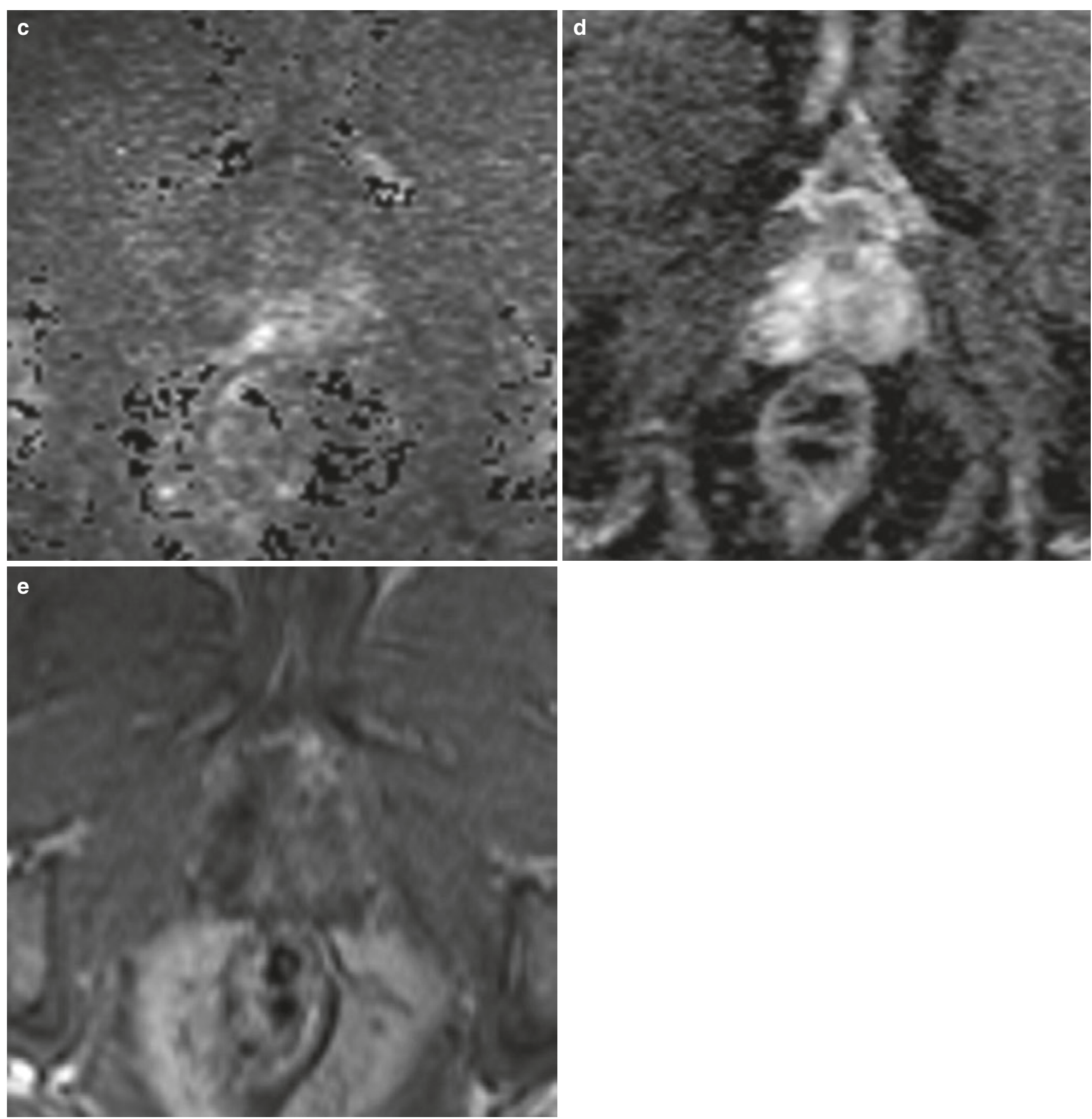

Fig. 11.3 (continued) 

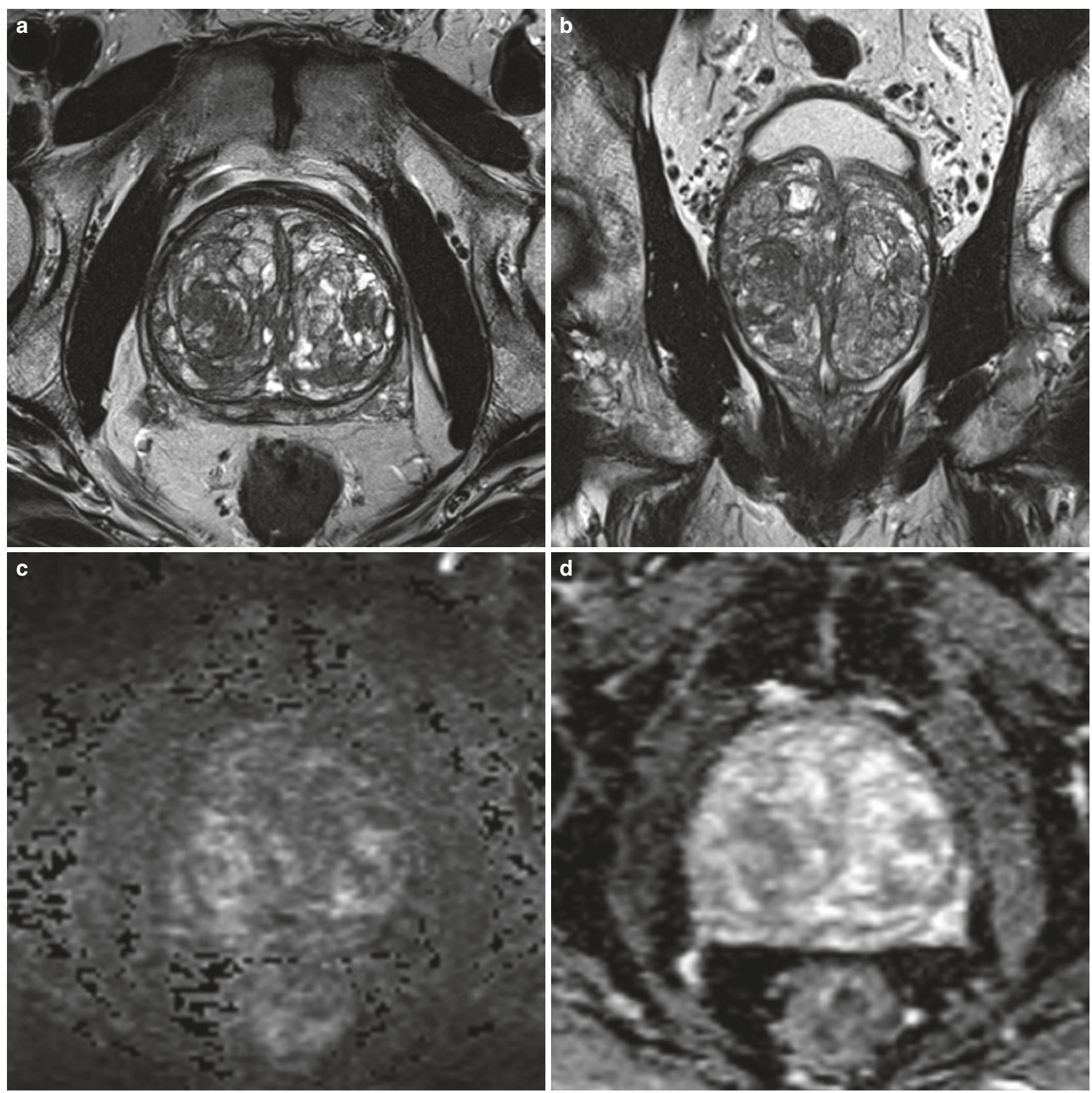

Fig. 11.4 (a-d) PI-RADS 2 findings of the transition zone in a patient with benign prostatic hyperplasia $(\mathrm{BPH})$. (a) Axial and (b) coronal T2-weighted sequence (dominant sequence for the transition zone) showing multiple circumscribed heterogeneous encapsulated nodules

(dark T2-rim) within the enlarged transition zone (overall PI-RADS score 2). (c) Diffusion-weighted high $b$-value (calculated $b=1400 \mathrm{~s} /$ $\mathrm{mm}^{2}$ ) image shows no focal areas of moderately increased signal intensity. (d) ADC map shows no focal hypointense areas (PI-RADS score 2) 

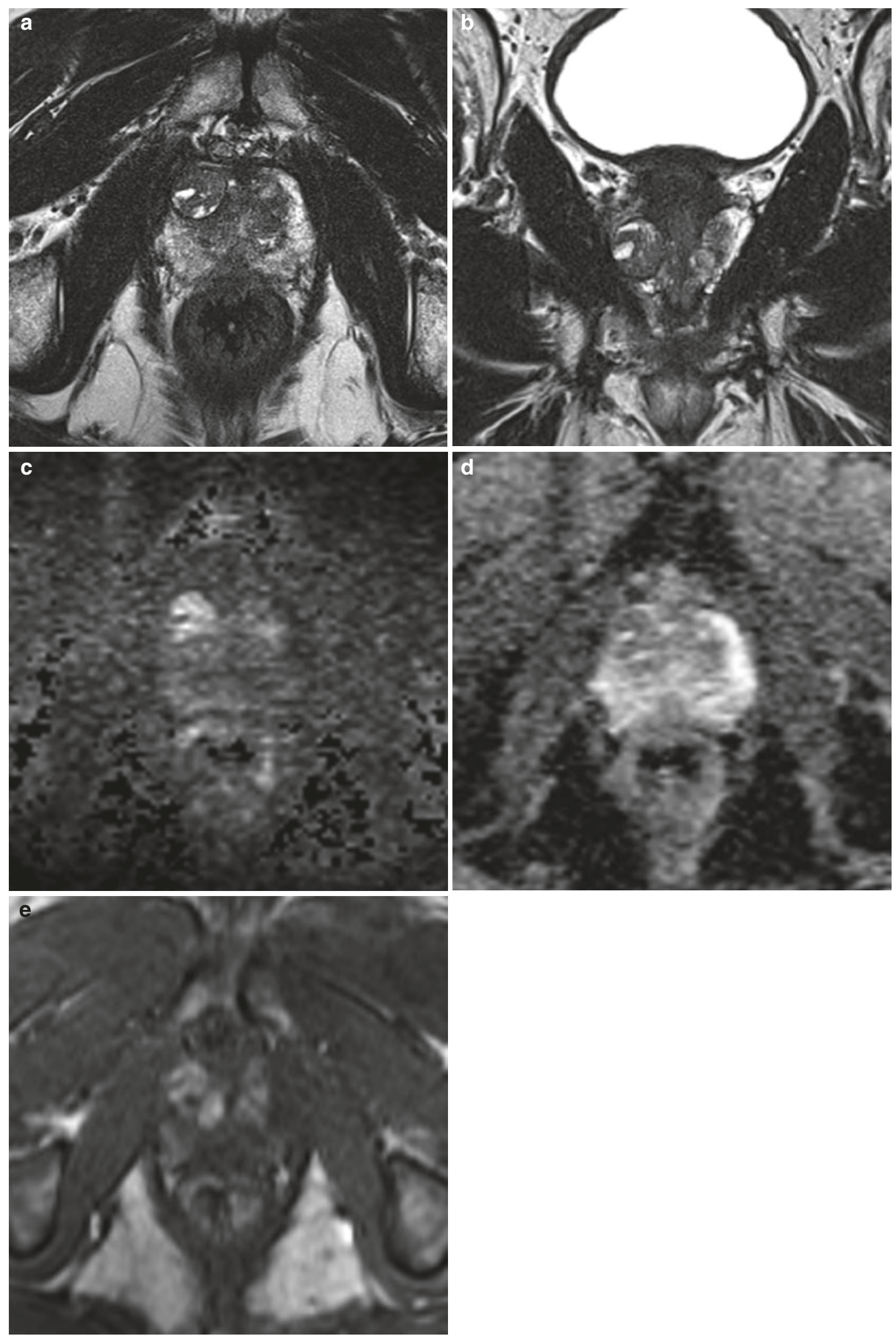

Fig. 11.5 (a-e) Protruded BPH node in the right anterior gland. (a) Axial and (b) coronal T2-weighted sequence (dominant sequence for the transition zone) showing a circumscribed heterogeneous encapsulated nodule (overall PI-RADS score 2). (c) Diffusion-weighted high $b$-value (calculated $b=1400 \mathrm{~s} / \mathrm{mm}^{2}$ ) image shows increased signal intensity. (d)
ADC map shows a focal hypointensity which is related to stromal $\mathrm{BPH}$ components which corresponds to the BPH nodule (therefore PI-RADS score of 2). (e) DCE sequence showing focal enhancement which corresponds to the lesion that demonstrates clear features of a BPH node (therefore DCE negative) consistent with an overall PI-RADS score of 2 

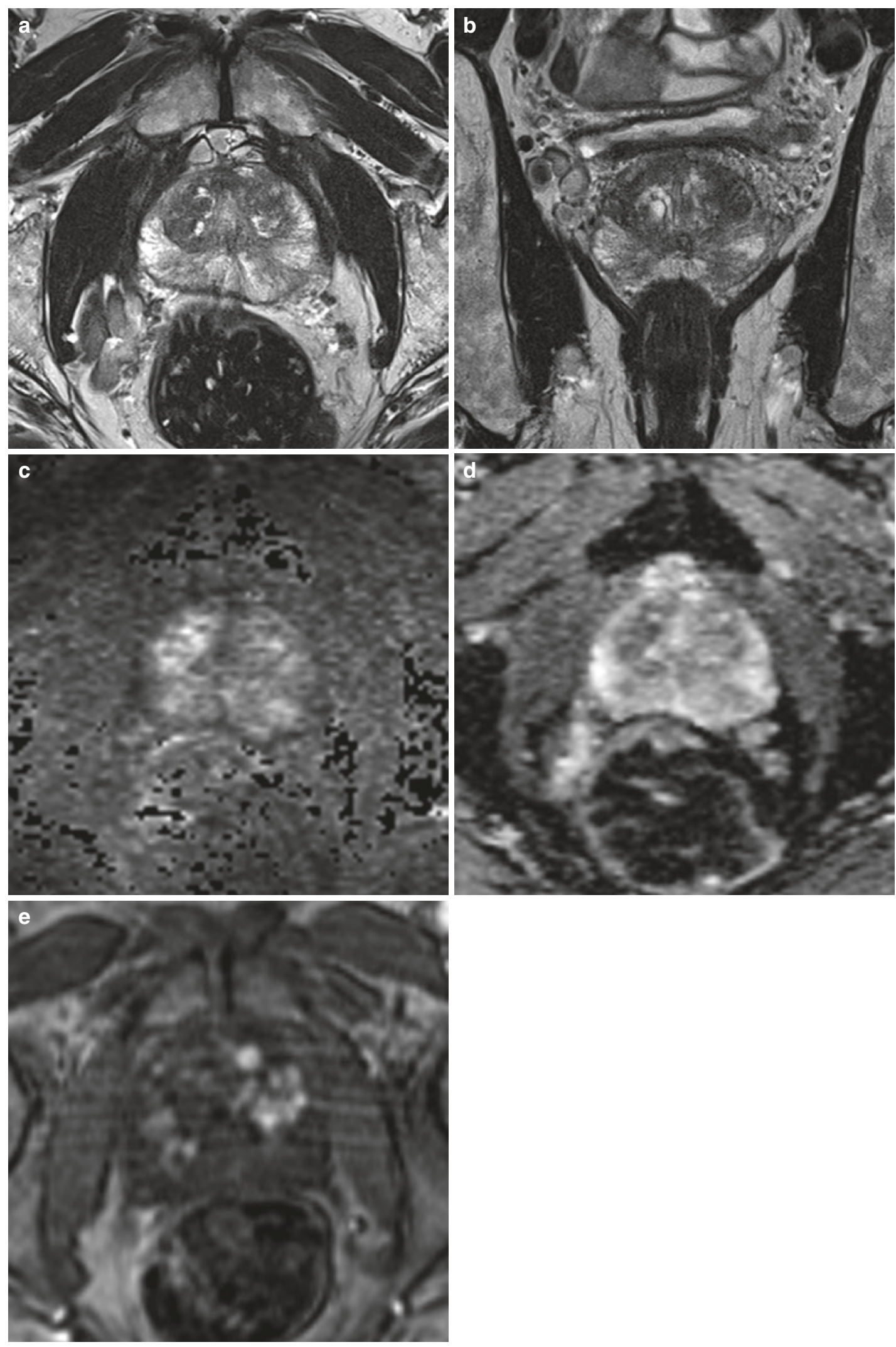

Fig.11.6 (a-e) PI-RADS 3 changes of the peripheral zone. (a) Axial and (b) coronal $\mathrm{T} 2$-weighted sequence showing heterogeneous noncircumscribed changes of the bilateral peripheral zone (PI-RADS score 3 ). (c) Diffusion-weighted high $b$-value (calculated $b=1400 \mathrm{~s} / \mathrm{mm}^{2}$ ) image shows a mildly hyperintense signal intensity. (d) ADC map (dominant sequence for the peripheral zone) shows moderately hypointense changes in the bilateral peripheral zone (PI-RADS score 3). (e) DCE sequence shows no focal early enhancement (DCE negative) consistent with an overall PI-RADS score of 3. Randomized TRUS-guided prostate biopsy revealed mild chronic prostatitis with no evidence for prostate cancer 

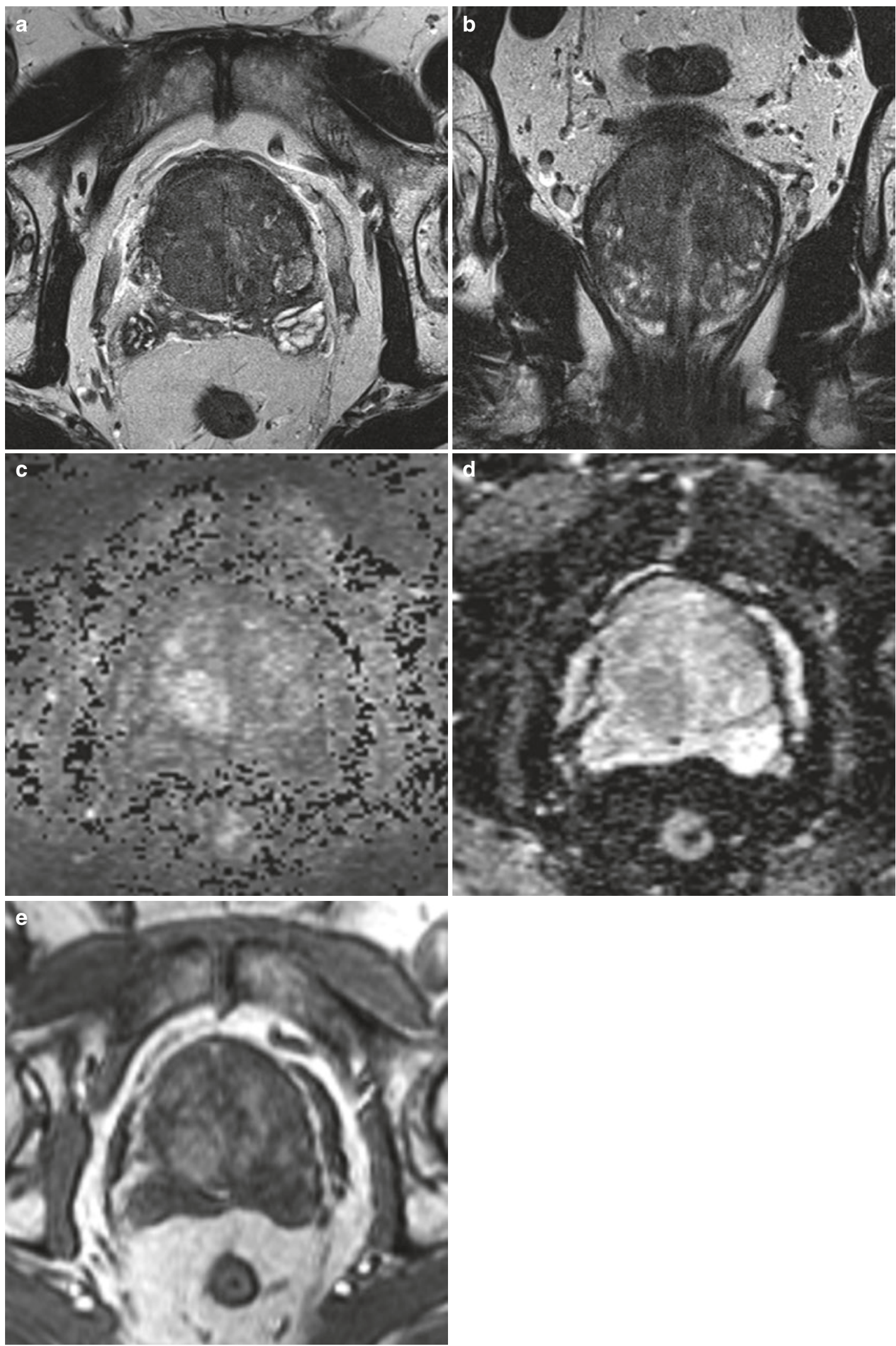

Fig. 11.7 (a-e) PI-RADS 3 changes of the transition zone in a patient with benign prostatic hyperplasia (BPH). (a) Axial and (b) coronal $\mathrm{T} 2$-weighted sequence (dominant sequence for the transition zone) showing heterogeneous signal intensity with obscured margins within the enlarged transition zone (overall PI-RADS score 3). (c) Diffusionweighted high $b$-value (calculated $b=1400 \mathrm{~s} / \mathrm{mm}^{2}$ ) image shows mildly hyperintense signal intensity. (d) ADC map shows moderately hypointense areas (PI-RADS score 3). (e) DCE sequence shows diffuse enhancement not corresponding to a focal finding on any other sequence (DCE negative). Randomized TRUS-guided biopsy revealed no malignancy; therefore, the findings can be attributed to BPH with predominantly stromal ( $\mathrm{T} 2$ hypointense) components 

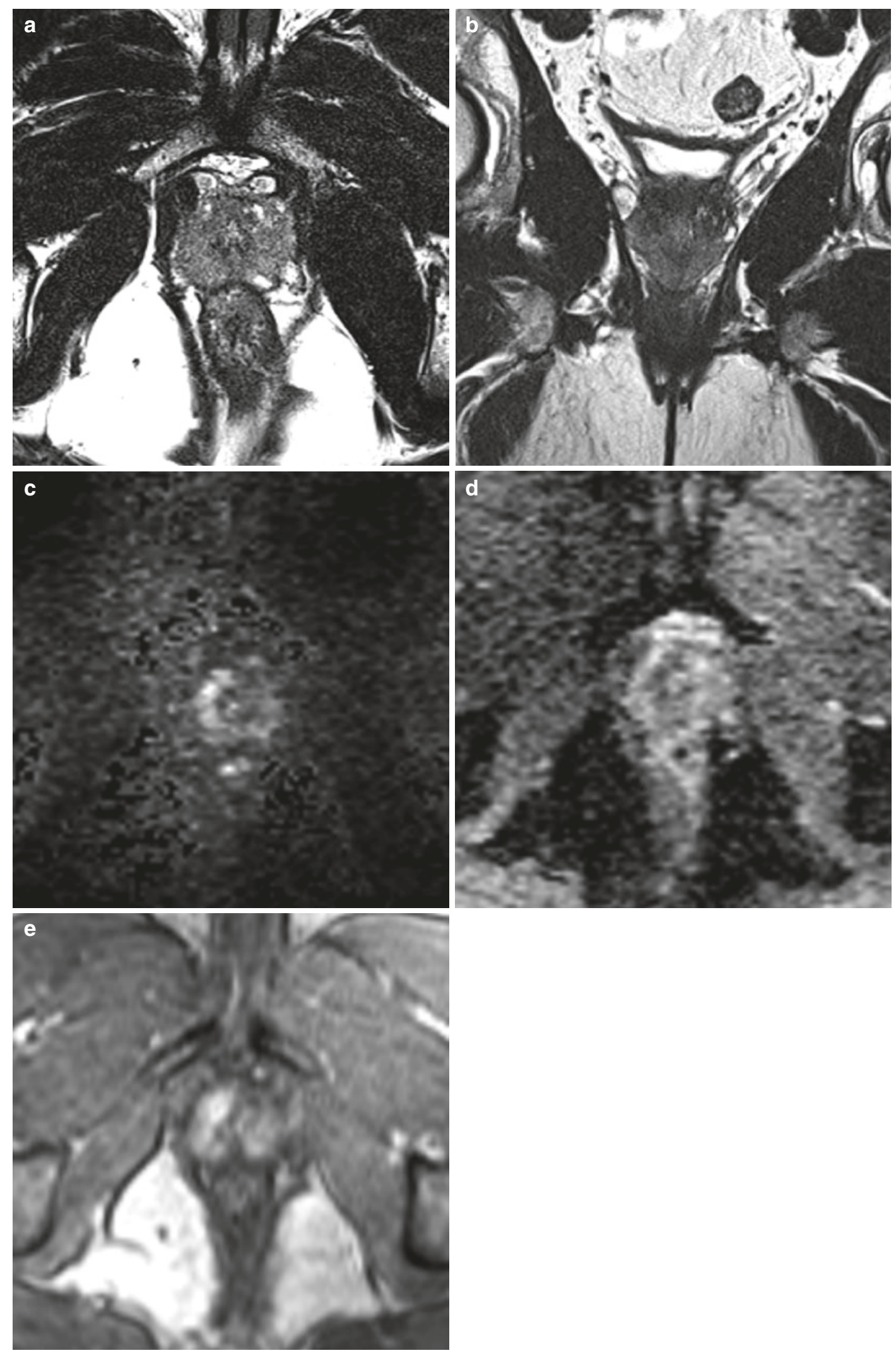

Fig. 11.8 (a-e) PI-RADS 4 lesion in the peripheral zone. (a) Axial and (b) coronal T2-weighted sequence showing moderate diffuse (noncircumscribed) hypointensity of the bilateral peripheral zone (PI-RADS score 3). (c) Diffusion-weighted high $b$-value (calculated $b=1400 \mathrm{~s} /$ $\mathrm{mm}^{2}$ ) image shows mildly hyperintense signal intensity in the right anterior and lateral peripheral zone (PI-RADS score 3). (d) ADC map cor- respondingly shows moderate hypointense signal intensity (dominant sequence for the peripheral zone) in the right anterior and lateral peripheral zone (PI-RADS score 3). (e) DCE sequence shows focal and contemporary enhancement (DCE positive) consistent with an upgrading to an overall PI-RADS score of 4 . MRI/US fusion-guided biopsy revealed a Gleason $3+4=7$ adenocarcinoma (PSA level was $6.9 \mathrm{ng} / \mathrm{mL}$ ) 

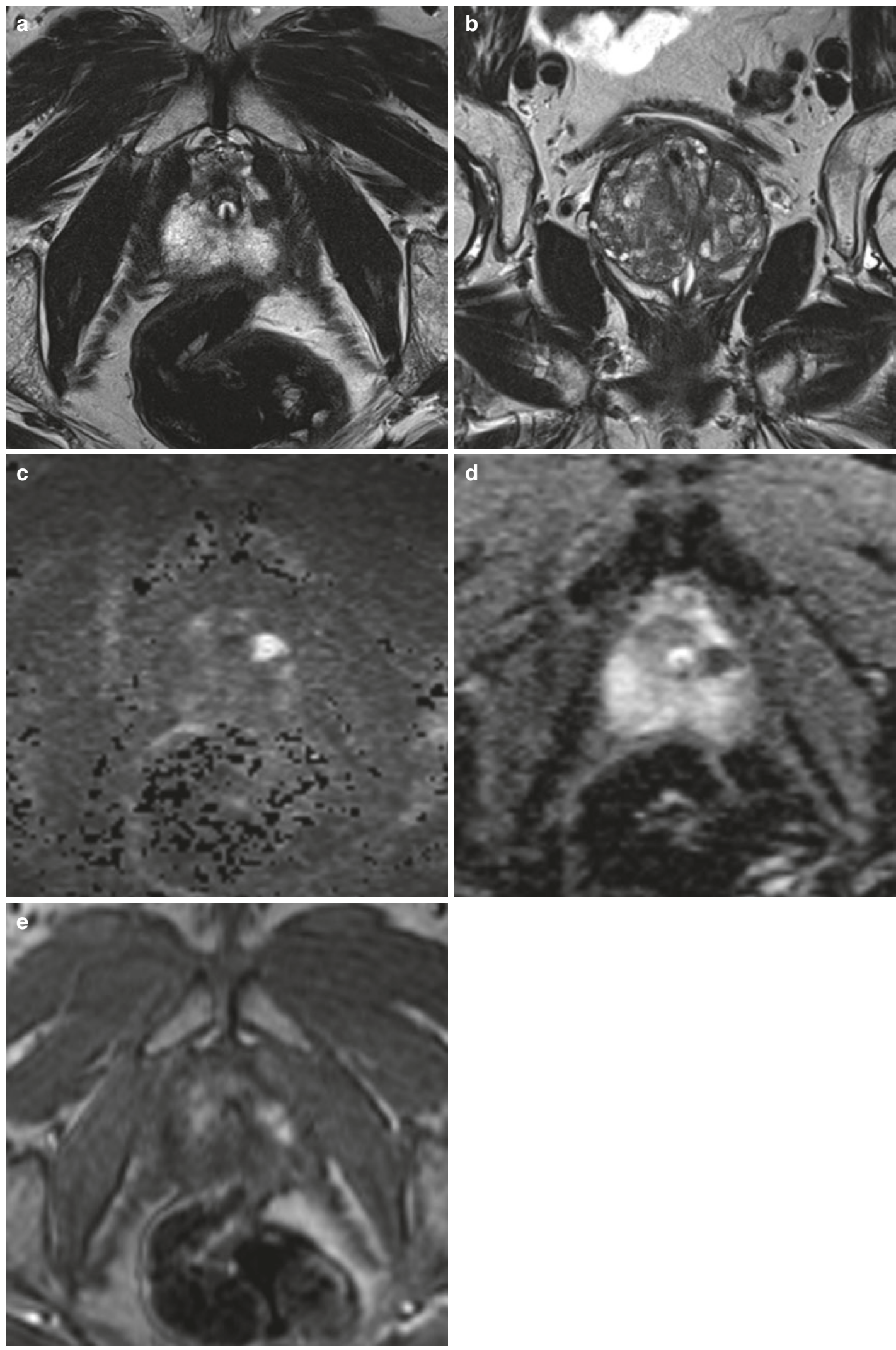

Fig.11.9 (a-e) PI-RADS 4 lesion in the peripheral zone. (a) Axial and (b) coronal T2-weighted sequence showing a circumscribed $11 \mathrm{~mm}$ hypointense mass in the left lateral peripheral zone (PI-RADS score 4). (c) Diffusion-weighted high $b$-value (calculated $b=1400 \mathrm{~s} / \mathrm{mm}^{2}$ ) image shows focal markedly hyperintense signal intensity of the mass. (d) ADC map (dominant sequence for the peripheral zone) correspond-

ingly shows focal markedly hypointense signal intensity of the mass (PI-RADS score 4). (e) DCE sequence shows focal and early enhancement (DCE positive) corresponding to the focal mass seen on T2-weighted and DWI consistent with an overall PI-RADS score of 4. MRI/US fusion-guided biopsy revealed a Gleason $4+3=7$ adenocarcinoma (PSA level was $8.1 \mathrm{ng} / \mathrm{mL}$ ) 

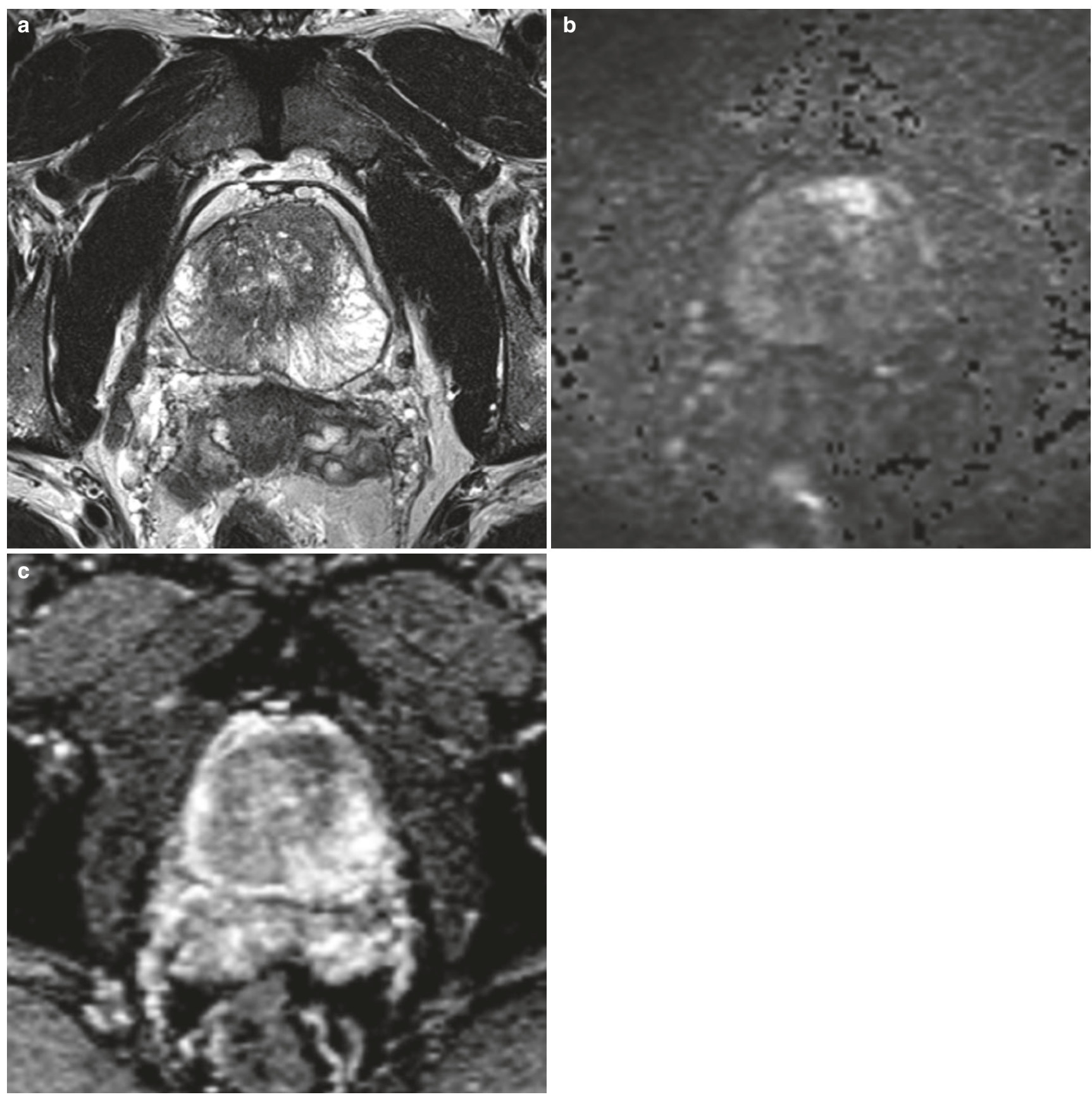

Fig. 11.10 (a-c) PI-RADS 4 lesion in the transition zone. (a) Axial T2-weighted sequence (dominant sequence for the transition zone) showing a circumscribed lenticular $14 \mathrm{~mm}$ hypointense mass in the left anterior transition zone with bulging of the prostatic capsule (PI-RADS score 4). (b) Diffusion-weighted high $b$-value (calculated $b=1400 \mathrm{~s} /$ $\mathrm{mm}^{2}$ ) image shows focal markedly hyperintense signal intensity of the mass. (c) ADC map correspondingly shows focal markedly hypointense signal intensity of the mass (PI-RADS score 4). MRI/US fusion-guided biopsy revealed a Gleason $4+4=8$ adenocarcinoma (PSA level was $9.8 \mathrm{ng} / \mathrm{mL}$ ). The patient had undergone a randomized TRUS-guided biopsy 3 months earlier with no evidence for malignancy. The anterior location is typical for adenocarcinoma missed by randomized biopsy; therefore, MRI is particularly useful in patients with negative randomized biopsy and persisting clinical suspicion for prostate cancer 

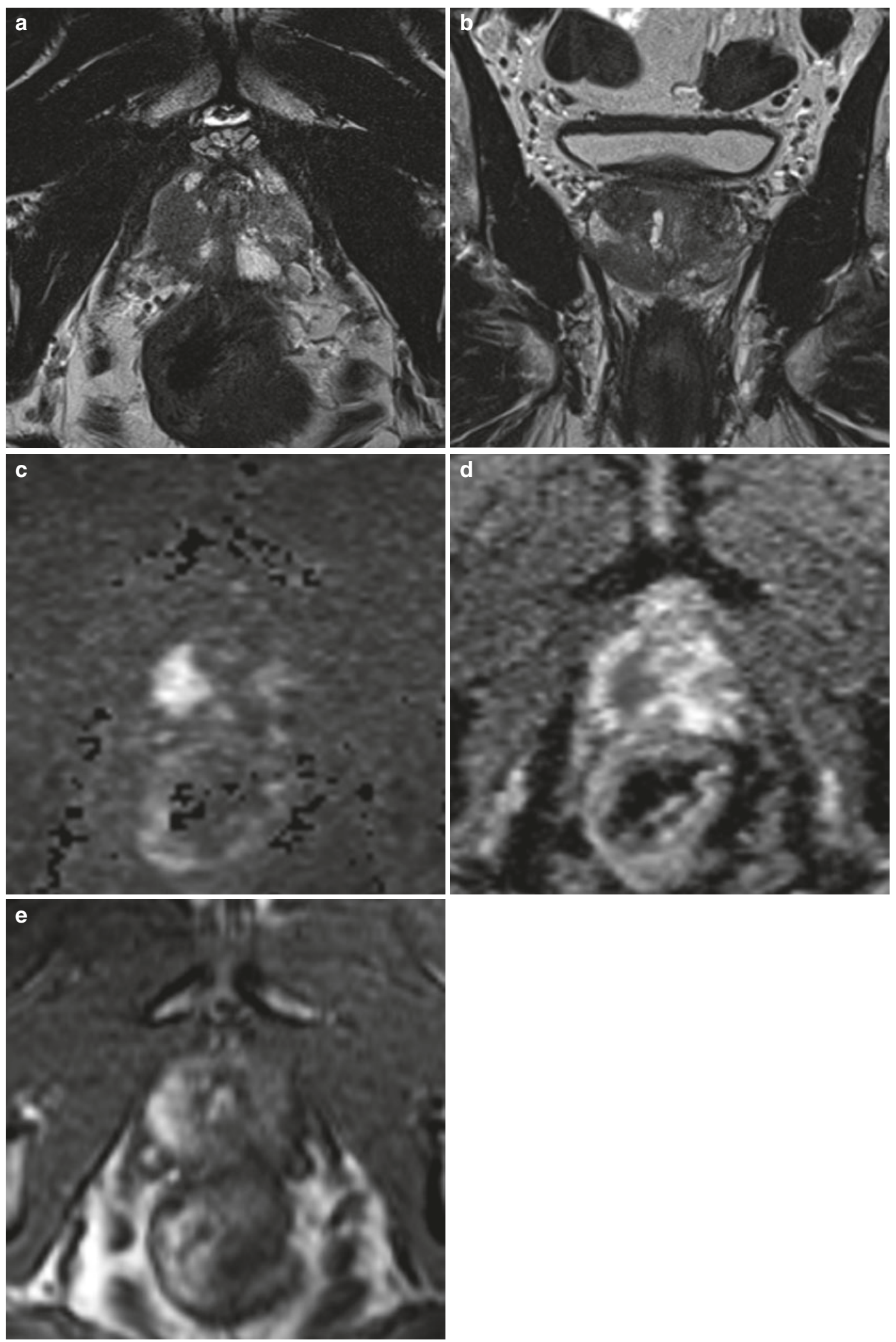

Fig. 11.11 (a-e) PI-RADS 5 lesion in the peripheral zone. (a) Axial and (b) coronal T2-weighted sequence showing a circumscribed $18 \mathrm{~mm}$ hypointense mass in the right lateral peripheral zone (PI-RADS score 5). (c) Diffusion-weighted high $b$-value (calculated $b=1400 \mathrm{~s} / \mathrm{mm}^{2}$ ) image shows focal markedly hyperintense signal intensity of the mass. (d) ADC map (dominant sequence for the peripheral zone) correspondingly shows focal markedly hypointense signal intensity of the mass (PI-RADS score 5). (e) DCE sequence shows focal and early enhance- ment (DCE positive) corresponding to the focal mass seen on T2-weighted and DWI consistent with an overall PI-RADS score of 5. MRI/US fusion-guided biopsy revealed a Gleason $4+3=7$ adenocarcinoma (PSA level was $11.2 \mathrm{ng} / \mathrm{mL}$ ). Also note the wedge-shaped T2 hypointensities in the left lateral peripheral zone which demonstrate moderately hypointense signal on the ADC map and no focal enhancement (PI-RADS 3). On biopsy multifocal prostate cancer was diagnosed with Gleason $3+3=6$ pattern in the left prostatic lobe 

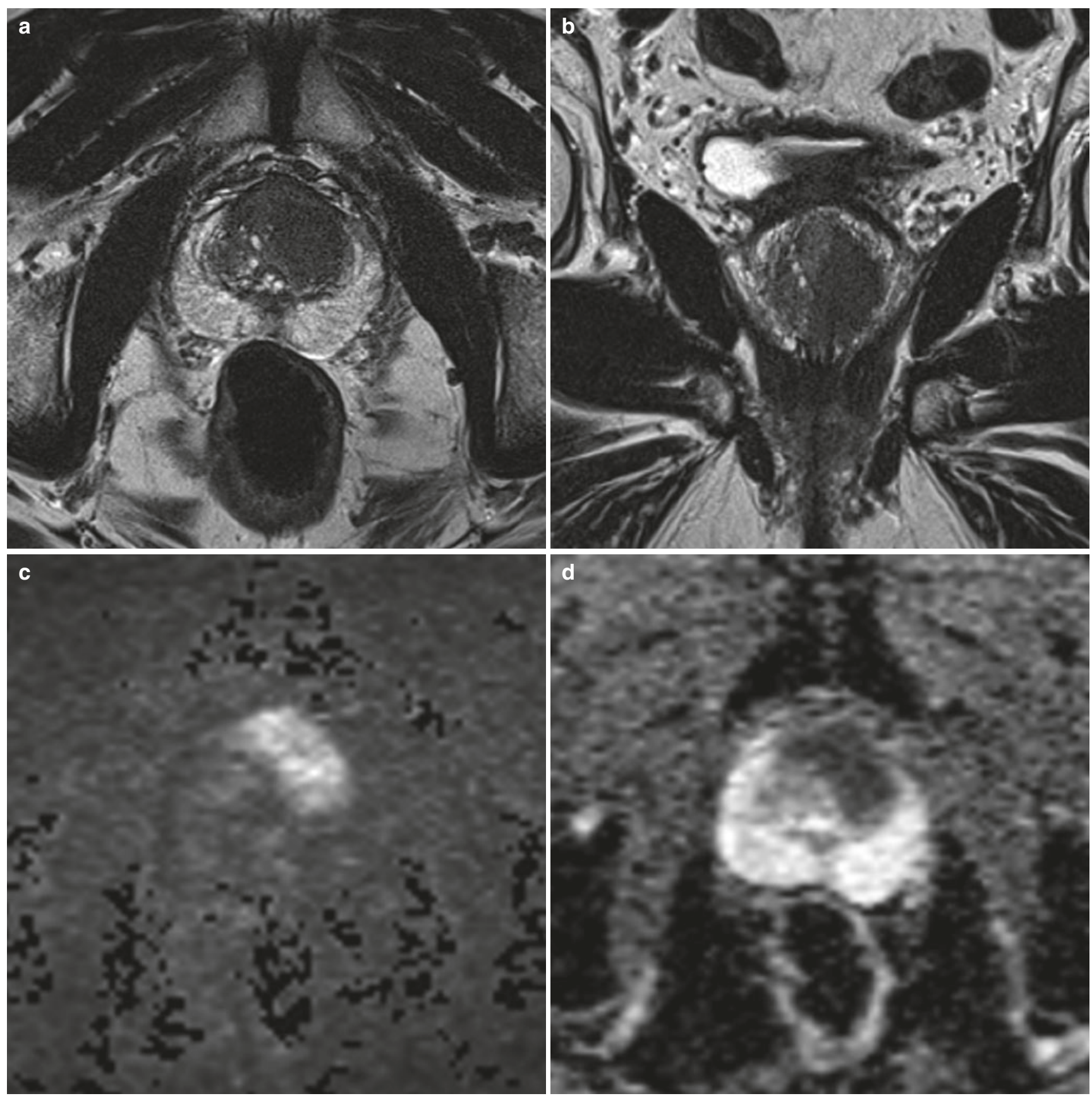

Fig. 11.12 (a-d) PI-RADS 5 lesion in the transition zone. (a) Axial and (b) coronal T2-weighted sequence (dominant sequence for the transition zone) showing a circumscribed lenticular $20 \mathrm{~mm}$ hypointense mass in the anterior transition zone with minimal bulging of the prostatic capsule (PI-RADS score 5). (c) Diffusion-weighted high $b$-value

(calculated $b=1400 \mathrm{~s} / \mathrm{mm}^{2}$ ) image shows focal markedly hyperintense signal intensity of the mass. (d) ADC map correspondingly shows focal markedly hypointense signal intensity of the mass (PI-RADS score 4). MRI/US fusion-guided biopsy revealed a Gleason $4+3=7$ adenocarcinoma (PSA level was $14.7 \mathrm{ng} / \mathrm{mL}$ ) 

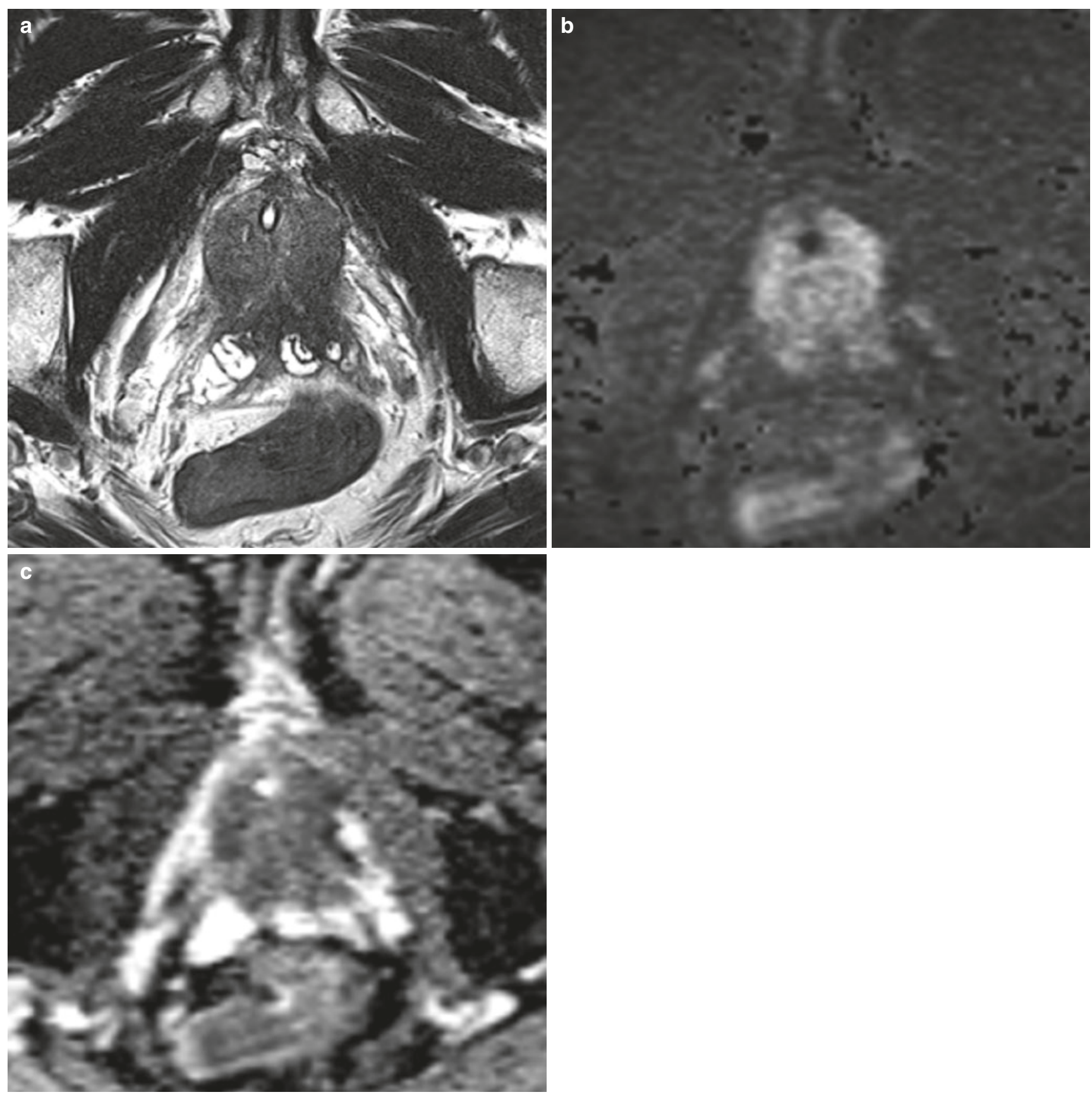

Fig. 11.13 (a-c) Locally advanced prostate cancer with seminal vesicle infiltration. (a) Axial T2-weighted sequence showing diffuse hypointensity of the entire prostate (zonal anatomy not visible) with extension into the bilateral seminal vesicles (PI-RADS score 5). (b) Diffusion-weighted high $b$-value (calculated $b=1400 \mathrm{~s} / \mathrm{mm}^{2}$ ) image

shows markedly hyperintense signal intensity of the entire prostate. (c) ADC map correspondingly shows markedly hypointense signal intensity of the prostate (PI-RADS score 5). Randomized TRUS-guided biopsy revealed a Gleason $4+5=9$ adenocarcinoma (PSA level was $26.5 \mathrm{ng} / \mathrm{mL})$ 
Table 11.5 Peripheral zone (PZ)

\begin{tabular}{|l|l|l|l|}
\hline DWI & T2 & DCE & PI-RADS score \\
\hline 1 & Any & Any & 1 \\
\hline 2 & Any & Any & 2 \\
\hline 3 & Any & Negative & 3 \\
\hline 4 & Positive & 4 \\
\hline 5 & Any & Any & 4 \\
\hline
\end{tabular}

Table 11.6 Transition zone (TZ)

\begin{tabular}{|l|l|l|l|}
\hline T2 & DWI & DCE & PI-RADS score \\
\hline 1 & Any & Any & 1 \\
\hline 2 & Any & Any & 2 \\
\hline 3 & $\leq 4$ & Any & 3 \\
\hline 4 & 5 & & 4 \\
\hline 5 & Any & Any & 4 \\
\hline
\end{tabular}

been introduced. The dominant sequence depends on the prostatic zone the lesion is located; therefore identification of the zonal anatomy is crucial. The area at the base of the prostate where the central zone borders the peripheral zone and the anterior gland where the anterior horn of the peripheral zone borders the transition zone and the anterior fibromuscular stroma might be challenging in this respect. The DWI sequence is the dominant sequence for the peripheral zone where most prostate cancers are located. The T2-weighted sequence is the dominant sequence for the transition zone. The dominant sequence defines the final PI-RADS score with the exception of PI-RADS 3 lesions, where for the peripheral zone the DCE sequence and for the transition zone the DWI sequence defines the final PI-RADS score (see Tables 11.5 and 11.6).

If a particular sequence cannot be acquired or is nondiagnostic due to artifacts (e.g., DWI when certain hip implants are present), it should be marked with "x." In this situation, the following rules apply:

Assessment without DWI (applies to PZ and TZ): the T2-weighted sequences define the final PI-RADS score with the exception of PI-RADS 3 -if the lesion is DCE negative, the final score remains 3; if the lesion is DCE positive, the final score is 4.

Assessment without DCE (only applies to the peripheral zone since DCE is not needed for transition zone scoring): the DWI score represents the final PI-RADS score.

\subsubsection{Structured Reporting}

A very important task of PI-RADS is to "simplify and standardize the terminology and content of radiology reports" and to "enhance interdisciplinary communications with referring clinicians." A comprehensive mpMRI report should therefore include the following contents:
The volume of prostate should be reported according to the ellipsoid formula: maximum AP diameter $\mathrm{x}$ maximum transverse diameter $x$ maximum longitudinal diameter $\times 0.52$.

The aforementioned PI-RADS score is assigned to up to four intraprostatic lesions with a score $\geq 3$. In case of multiple lesions, an index lesion should be defined. The index lesion is the one with the highest PI-RADS score. In case multiple lesions qualify for the highest PI-RADS score, extraprostatic extension (EPE) outclasses lesion size. For each lesion a PI-RADS score is assigned to the series and the image number where the lesion is best visualized should be reported to assist the urologist in selecting optimal images for MRI/US fusion-guided prostate biopsy. The lesion size also needs to be reported. Measurement of each lesion is preferred on the axial images; the DWI sequence should be used for peripheral zone lesions and the T2-weighted images for transition zone lesions. If a lesion is not well delineated on the axial sequences, then another plain should be used.

Another crucial element of a full PI-RADS report is a sector map in which the lesions should be indicated, since this particularly enhances the communication with the urologist. For this matter, the prostate is subdivided into three axial heights, the base, the midgland, and the prostatic apex, respectively. The seminal vesicles should also be included for cases of extraprostatic extension. The zonal anatomy (peripheral zone, transition zone, central zone, and anterior fibromuscular stroma) and the urethra should also be incorporated into the sector map.

\subsection{Concluding Remarks}

Comprehensive multiparametric MRI of the prostate should include lesion scoring and reporting according to the PI-RADS system. This will assist to achieve a high level of diagnostic accuracy and assure a thriving communication with the urologist.

\section{Key Points}

- Continuing technical innovation and broad research activity have resulted in a high diagnostic accuracy of MRI of prostate.

- Prostate MRI plays an important role for imagingbased targeted prostate biopsy.

- In patients undergoing active surveillance of prostate carcinoma, MRI has become indispensable for initial assessment as well as during the surveillance of the disease.

- PI-RADS standardizes image acquisition and facilitates communication with the urologists. It is considered an obligatory key element in prostate MRI.

- Comprehensive multiparametric MRI of the prostate should include lesion scoring and reporting according to the PI-RADS system. 


\section{Suggested Reading}

Ahmed HU, El-Shater Bosaily A, Brown LC, PROMIS Study Group, et al. Diagnostic accuracy of multi-parametric MRI and TRUS biopsy in prostate cancer (PROMIS): a paired validating confirmatory study. Lancet. 2017;389:815-22.

Barentsz JO, Richenberg J, Clements R, European Society of Urogenital Radiology, et al. ESUR prostate MR guidelines 2012. Eur Radiol. 2012;22:746-57.

Cash H, Günzel K, Maxeiner A, et al. Men with a negative real-time MRI/ultrasound-fusion guided targeted biopsy but prostate cancer detection on TRUS-guided random biopsy - what are the reasons for targeted biopsy failure? BJU Int. 2016;118:35-43.

Cash H, Maxeiner A, Stephan C, et al. The detection of significant prostate cancer is correlated with the prostate imaging reporting and data system (PI-RADS) in MRI/transrectal ultrasound fusion biopsy. Word J Urol. 2016;34:525-32.

Greer MD, Shih JH, Lay N, et al. Validation of the dominant sequence paradigm and role of dynamic contrast-enhanced imaging in PI-RADS version 2. Radiology. 2017;285(3):859-69.
Haas M, Günzel K, Penzkofer T, et al. Implications of PI-RADS version 1 and updated version 2 on the scoring of prostatic lesions on multiparametric MRI. Aktuelle Urol. 2016;47:383-7.

http://www.esur.org/esur-guidelines/prostate-mri/. Accessed 4 Aug 2017.

Polanec S, Helbich TH, Bickel H, et al. Head-to-head comparison of PI-RADS v2 and PI-RADS v1. Eur J Radiol. 2016;85:1125-31.

Purysko AS, Bittencourt LK, Bullen JA, et al. Accuracy and interobserver agreement for Prostate Imaging Reporting and Data System, Version 2 , for the characterization of lesions identified on multiparametric MRI of the prostate. AJR Am J Roentgenol. 2017;209:339-49.

Rosenkrantz AB, Ginocchio LA, Cornfeld D, et al. Interobserver reproducibility of the PI-RADS version 2 lexicon: a multicenter study of six experienced prostate radiologists. Radiology. 2016;280:793-804.

Ullrich T, Quentin M, Oelers C, et al. Magnetic resonance imaging of the prostate at 1.5 versus $3.0 \mathrm{~T}$ : a prospective comparison study of image quality. Eur J Radiol. 2017;90:192-7.

Weinreb JC, Barentsz JO, Choyke PL, et al. PI-RADS prostate imaging - reporting and data system: 2015, version 2. Eur Urol. 2015;69:16-40.

Open Access This chapter is licensed under the terms of the Creative Commons Attribution 4.0 International License (http://creativecommons.org/ licenses/by/4.0/), which permits use, sharing, adaptation, distribution and reproduction in any medium or format, as long as you give appropriate credit to the original author(s) and the source, provide a link to the Creative Commons license and indicate if changes were made.

The images or other third party material in this book are included in the book's Creative Commons license, unless indicated otherwise in a credit line to the material. If material is not included in the book's Creative Commons license and your intended use is not permitted by statutory regulation or exceeds the permitted use, you will need to obtain permission directly from the copyright holder. 